\title{
Similarity and contrasts between thermodynamic properties at the critical point of liquid alkali metals and of electron-hole droplets
}

\author{
F.E. Leys, ${ }^{1}$ N.H. March, ${ }^{1,2}$ G.G.N. Angilella, ${ }^{3}$ and M.-L. Zhang ${ }^{4}$ \\ ${ }^{1}$ Physics Department, Antwerp University (RUCA), Groenenborgerlaan 171, B-2020 Antwerpen, Belgium \\ ${ }^{2}$ Oxford University, Oxford, England \\ ${ }^{3}$ Dipartimento di Fisica e Astronomia, Università di Catania, \\ and Istituto Nazionale per la Fisica della Materia, UdR di Catania, Via S. Sofia, 68, I-95123 Catania, Italy \\ ${ }^{4}$ Chemical Physics Department, Weizmann Institute of Science, Rehovot 76100 Israel \\ (Received 27 February 2002; revised manuscript received 23 May 2002; published 30 August 2002)
}

\begin{abstract}
The recent experimental study by means of time-resolved luminescence measurements of an electron-hole liquid (EHL) in diamond by Shimano et al. [Phys. Rev. Lett. 88, 057404 (2002)] prompts us to compare and contrast critical temperature $T_{c}$ and critical density $n_{c}$ relations in liquid alkali metals with those in electronhole liquids. The conclusion drawn is that these systems have similarities with regard to critical properties. In both cases the critical temperature is related to the cube root of the critical density. The existence of this relation is traced to Coulomb interactions and to systematic trends in the dielectric constant of the electron-hole systems. Finally a brief comparison between the alkalis and EHL's of the critical values for the compressibility ratio $Z_{c}$ is also given.
\end{abstract}

DOI: 10.1103/PhysRevB.66.073314

PACS number(s): 71.35.Ee, 71.30.+h

Two of us ${ }^{1}$ have been concerned in earlier work with the behavior of the thermodynamic quantities $T_{c}, n_{c}$, and $p_{c}$, at the critical points of the fluid alkali metals. Relationships were shown to exist between critical temperature $T_{c}$ and critical number density $n_{c}$ for the five fluid alkalis, the form anticipated in the earlier investigation of Chapman and March, ${ }^{2}$ namely

$$
T_{c} n_{c}^{-1 / 3}=\text { const, }
$$

being confirmed in Ref. 1 .

The present study has been prompted by the very recent experiment of Shimano et al., ${ }^{3}$ who report on the formation of an electron-hole liquid ${ }^{4}$ (EHL) with a high critical temperature $T_{c}=165 \mathrm{~K}$ in diamond, by means of time-resolved luminescence measurements under an intense femtosecond photoexcitation above the band gap. Then, by a timeresolved spectral shape analysis, a very high carrier density $n_{0}=1.0 \times 10^{20} \mathrm{~cm}^{-3}$ at $T=0$ is revealed, together with the high value of the critical temperature $T_{c}$ already recorded above.

In addition to the important findings for the EHL in diamond, Shimano et $a l^{3}$ comment in their introduction on the similarities between the physical properties of the EHL and liquefied metals. It is their comment which has motivated the present study, in which we compare and contrast EHL properties with those of the fluid alkali metals. Equation (1) above provides a natural starting point, but unfortunately while $T_{c}$ is known for at least 5 EHL's, the same is not true for the critical density $n_{c}$.

However, prompted by form (1), we have taken data from Ref. 3, and have collected $T_{c}$ and the zero temperature densities $n_{0}$ for five EHL's in Table I of the present paper. From these values, it can be readily shown that whereas $T_{c}$ increases by a factor $\sim 25$ in going from Ge to diamond, the zero-temperature quantity $n_{0}$ increases by a factor $4 \times 10^{2}$. Due to form (1), which we emphasize however contains the critical density $n_{c}$ in the fluid alkalis, we have studied first the question as to whether $T_{c}$ correlates with $n_{0}$ in the five EHL's referred to in Table I. After some numerical investigation, we have constructed Fig. 1, which demonstrates beyond a reasonable doubt that $T_{c}$ and $n_{0}$ indeed correlate strongly. There is a power-law behavior between $T_{c}$ and $n_{0}$, although the $\frac{1}{3}$ power in Eq. (1) must be modified (empirically to $\frac{1}{2}$ : see the caption to Fig. 1 and the further comments below). However, while Fig. 1 displays similarities of shape with Eq. (1) for the fluid alkalis, this equation contains the critical density $n_{c}$ whereas for lack of experimental information, Fig. 1 correlates $T_{c}$ with the zero-temperature density $n_{0}$.

In the absence of experimental information on $n_{c}$, we have sought a theoretical basis for bringing Eq. (1) and Fig. 1 into closer contact. We are considerably aided here by the work of Kalt et al. ${ }^{5}$ These authors observed picosecond electron-hole droplet formation in the indirect gap material $\mathrm{Al}_{x} \mathrm{Ga}_{1-x} \mathrm{As}$. In the course of their experimental work, they refer to a "scaling law"

$$
\frac{n_{c}}{n_{0}} \cong 0.3 \text {, }
$$

TABLE I. Values for the EHL number density $n_{0}$ at $T=0$, the critical temperature $T_{c}$, and the dielectric constant $\varepsilon$ in five indirect-gap semiconductors (extracted from Table I of Ref. 3).

\begin{tabular}{lccc}
\hline \hline & $n_{0}\left(\mathrm{~cm}^{-3}\right)$ & $T_{c}(\mathrm{~K})$ & $\epsilon$ \\
\hline $\mathrm{Ge}$ & $2.5 \times 10^{17}$ & 6.7 & 16.0 \\
$\mathrm{Si}$ & $3.3 \times 10^{18}$ & 24.5 & 12 \\
$\mathrm{Gap}$ & $6 \times 10^{18}$ & 40 & 9.1 \\
3C-SiC & $7.8 \times 10^{18}$ & 41 & 9.72 \\
Diamond & $1.0 \times 10^{20}$ & 165 & 5.7 \\
\hline \hline
\end{tabular}




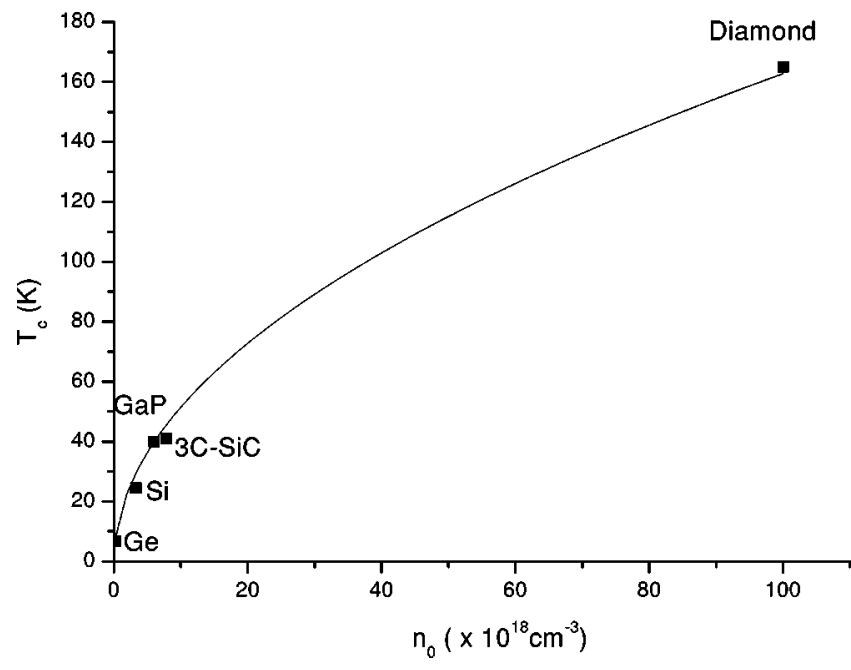

FIG. 1. Critical temperature $T_{c}$ of the EHL in the five semiconductors referred to in Table I vs the (zero temperature) number density $n_{0}$. The curve drawn is given by $T_{c} n_{0}^{-1 / 2}=16.3$ $\times 10^{-9} \mathrm{~cm}^{3 / 2} \mathrm{~K}$.

with a reference to Forchel et al. ${ }^{6}$ Obviously, if we adopt this so-called scaling law, then Eq. (1) and the result from Fig. 1 come into intimate context, and the conclusion is that there is a different exponent, say generally denoted by $\varkappa$, for the relationship

$$
T_{c} n_{c}^{-\varkappa}=\mathrm{const}
$$

with $\chi=\frac{1}{3}$ for the five fluid alkalis and $\sim \frac{1}{2}$ from Fig. 1 and Eq. (2) for the EHL's. Already, Chapman and March ${ }^{2}$ noted the totally different critical behavior for liquid alkali metals compared with condensed rare gases, for the latter case the exponent $x$ being -2 .

As this paper was nearing completion, we became aware of two further contributions. In the first of these, written more than 20 years ago by Reinecke and Ying, ${ }^{7}$ the authors had anticipated a relation $T_{c} n_{c}^{-1 / 2}=$ const. This is the more remarkable because it can be seen from the present Fig. 1 that the "diamond" point of Shimano et al. ${ }^{3}$ is crucial to fitting the parabolic form, and allowing the above constant to be made wholly quantitative. The writers who anticipated the parabolic relation, however, went on to express doubts as to whether such a relation had any fundamental basis. This leads us to the second contribution referred to above: that of Likal'ter. $^{8}$

Likal'ter studies what he emphasizes is the limiting situation of the EHL in which the hole mass $m_{h} \gg m_{e}$, the electron mass. He then gives individual formulas for the critical constants $T_{c}$ and $n_{c}$ discussed above, and also for the critical pressure $p_{c}$. Our interest in Likal'ter's formulas is to expose relations between these critical constants which can then be compared and contrasted with known results for the fluid alkali metals.

The first $T_{c}-n_{c}$ relation which follows from Likal'ter's model is

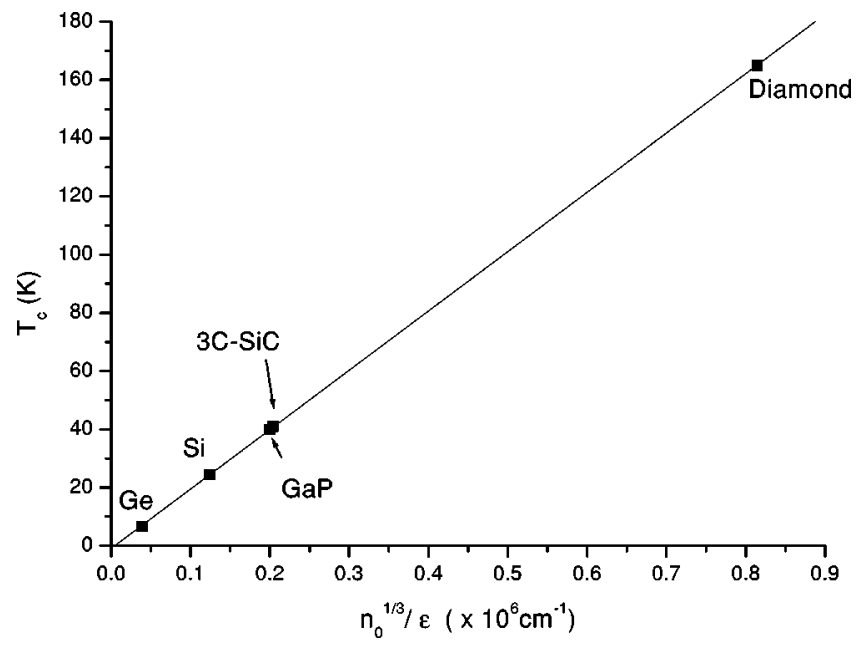

FIG. 2. Critical temperature $T_{c}$ of the EHL of five semiconductors referred to in Table I vs $n_{0}^{1 / 3} / \varepsilon$, where $n_{0}$ is the number density of the EHL at $T=0$ and $\varepsilon$ the dielectric constant.

$$
T_{c}=\left[\frac{4}{7}\right]^{2}\left[\frac{4 \pi}{3}\right]^{1 / 3} \frac{e^{2} \gamma}{2} \frac{n_{c}^{1 / 3}}{\varepsilon},
$$

where $\gamma$ is a renormalized Madelung constant, given by Likal'ter as $\approx 0.55$. This formula differs qualitatively from the fluid metals finding of Chapman and March ${ }^{2}$ by the dielectric constant $\varepsilon$ of the semiconductors appearing in the denominator. The limiting prediction of Eq. (4) deduced from Likal'ter's model will now be brought into close contact with the plot in Fig. 1. Thus in Fig. 2 we have used the data from Table I, including the experimental dielectric constant values recorded in the final column, to plot $T_{c}$ vs $n_{0}^{1 / 3} / \varepsilon$. There is clearly a linear relation as predicted by Likal'ter's model. Accepting scaling relation (2), the parabolic fit of the data in Fig. 1 can be reconciled with the linearity of Fig. 2 provided $\varepsilon$ and $n_{0}$ are related, of course approximately, by a $\frac{1}{6}$ power law. However, at present we have no fundamental justification for a relation $\varepsilon n_{0}^{1 / 6}$ $=$ const, because, at least in principle, such a formula could contain the binding energy of excitons, or the effective electron mass.

Returning to the similarities and differences from the fluid metals, we have also employed Likal'ter's individual formulas for $T_{c}, n_{c}$, and $p_{c}$ to calculate the so called compressibility ratio $Z_{c}$ defined as

$$
Z_{c}=\frac{p_{c}}{n_{c} k_{B} T_{c}}
$$

which was studied in detail for the five fluid alkalis in Ref. 1. The limiting formulas (for $m_{h} \gg m_{e}$ ) then lead to the result

$$
Z_{c}=\frac{7}{24}
$$

This is near to the value for the heavier alkalis, 0.217 for $\mathrm{Rb}$ and 0.203 for $\mathrm{Cs}$, but there is a wide variation through the alkali fluids, Li having a value of 0.064. The value in Eq. (6) 
is not so much smaller than the prediction from the van der Waals equation of state prediction, namely $3 / 8 .^{9}$

The conclusion is, first, that there are some interesting similarities between the EHL's and the fluid alkali metals with regard to critical properties. In both cases $T_{c}$ is related to $n_{c}^{1 / 3}$, which reflects the fingerprints of Coulomb interactions. For the EHL, however, in the limiting case studied by Likal'ter ${ }^{8}$ where $m_{h} \gg m_{e}$, this quantity $n_{c}^{1 / 3}$ is divided by the dielectric constant $\varepsilon$ of the semiconductors, high for $\mathrm{Si}$ and considerably reduced for diamond. Second, the limiting for- mulas of Likal'ter for $T_{c}, n_{c}$, and $p_{c}$ are shown to yield the constant critical compressiblility ratio $Z_{c}$ in Eq. (6), in contrast to the wide spread of values of $Z_{c}$ in the fluid alkalis.

N.H.M. wishes to acknowledge some financial support from the Francqui Foundation. Thanks are also due to the Executive Director, Professor L. Eyckmanns, for his help and encouragement. G.G.N.A thanks the University of Antwerp (RUCA) for much hospitality. Finally, the authors wish to acknowledge the constructive comments made by two referees, which have enabled us to improve the presentation of this paper.
${ }^{1}$ F.E. Leys, N.H. March, V.E. Van Doren, and G. Straub, Solid State Commun. 113, 479 (2000).

${ }^{2}$ R.G. Chapman and N.H. March, Phys. Chem. Liq. 16, 77 (1986).

${ }^{3}$ R. Shimano, M. Nagai, K. Horiuch, and M. Kuwata-Gonokami, Phys. Rev. Lett. 88, 057404 (2002).

${ }^{4}$ See, e.g., Electron-Hole Droplets in Semiconductors, edited by C. D. Jeffries and L.V. Keldysh (North-Holland, Amsterdam, 1983).
${ }^{5}$ H. Kalt, K. Reimann, W.W. Ruhle, M. Rinker, and E. Bauser, Phys. Rev. B 42, 7058 (1990).

${ }^{6}$ A. Forchel, B. Laurich, J. Wagner, W. Schmid, and T.L. Reinecke, Phys. Rev. B 25, 2730 (1982).

${ }^{7}$ T.L. Reinecke and S.C. Ying, Phys. Rev. Lett. 43, 1054 (1979).

${ }^{8}$ A.A. Likal'ter, Phys. Usp. 43, 777 (2000).

${ }^{9}$ See, e.g., N.H. March, Liquid Metals, Concepts and Theory (Cambridge University Press, Cambridge, 1990). 